\title{
Design of a Differential-Drive Wheeled Robot Controller with Pulse-Width Modulation
}

\author{
Farshad Arvin, Khairulmizam Samsudin, and M. Ali Nasseri
}

\begin{abstract}
This paper presents mobile robots motion control technique based on pulse-width modulation (PWM). This technique is employed on AMiR which is an autonomous miniature robot for swarm robotic platform. That uses differential drive with a caster wheel configuration. Robot's motors enable to work with different speed in different direction, forward and reverse. A microcontroller as the main processor is deployed to generate motor control pulses and manage duty-cycle of PWM signals. Two methods in robot trajectory control which are rotation and straight movement are described in this paper. Time estimation and also speed selection calculations illustrate the feasibility of this technique to be used in mobile robot motion control problem.
\end{abstract}

\section{INTRODUCTION}

$\mathrm{W}$ HEELED mobile robots are common platform for robotics research and education. All mobile robots need some wheels and motors to its trajectory. These use various motion configurations due to their applications. The motion of mobile robots must be modeled by mathematical calculation to estimate physical environment and executing defined scenarios.

Kinematical design of wheel-based robots are depend on application with they are applied [1,2]. Number of wheels and configuration of them needs a mathematical modeling which needs situation of real mechanical design outline, to have a reliable trajectory. Mobile robots use several classification of wheels situation such as differentially driven [3, 4], Car-type [5], Omni-directional [6, 7], and Synchro drive [8]. These wheels configurations need different controlling techniques in motion and trajectory.

One of common wheels configuration in mobile robot designs is the differential drive. In this technique, two motors are utilized to provide motion and one or two caster wheel(s) are used only for balancing robot's structure. Different speeds for wheels are used to achieve rotation and similar motor speed cause to straight motion. In fact, it is comfortable design for implementing mobile robots that are able to execute all defined trajectory. For implementing different control techniques, the flexible platform that enables to develop PWM commands is required.

For implementing proposed technique, a flexible mobile

Farshad Arvin and Khairulmizam Samsudin are with the Computer Systems Research Group, Department of Computer and Communication Systems Engineering, University of Putra Malaysia, UPM 43400, Serdang, Selangor, Malaysia (e-mail: gs21875@mutiara.upm.edu.my; kmbs@eng.upm.edu.my).

M. Ali Nasseri is with the Department of Mechatronic, Faculty of Electrical Engineering, Islamic Azad University, Qazvin, Iran (e-mail: a_nasseri@hotmail.com). robot as the experiments platform is deployed as shown in Fig. 1. It is designed for swarm mobile applications which utilized two small low-power geared DC motors to movement and one caster wheel in front to support structure. Proposed motion control technique does not need any changing in mechanical design and it is implemented with software programming. Setting PWM timers and selecting several duty-cycles according to decided behaviors is enough to achieve smooth motion for many wheeled mobile robots.

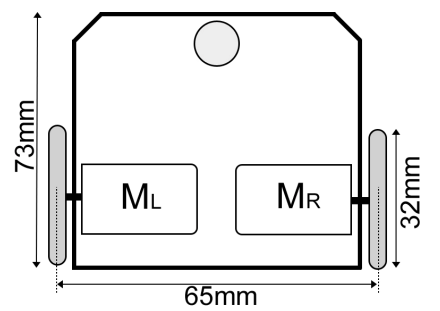

Fig.1 Physical dimension of the mobile robot prototype

The rest of this paper is organized as follows. Section II describes robot locomotion which includes DC motor, pulsewidth modulation technique, processing unit, and robot trajectory. The experimental results are presented in section III.

\section{ROBOT LOCOMOTION}

This section describes motion control techniques in robotic applications and also mechanical and hardware requirements to achieve smooth controllable mobile robot. Some important hardware and techniques such as motors and driving, variable speed methods, rotation techniques, and processing unit are described.

\section{A. DC Motor}

Direct-current (DC) motors are employed in many different robotic applications with various scales. According to the right-hand rule, the changing in current direction causes to change the direction of motion. DC motors' speed measured by RPM (rotate per minute) and it is calculated by the following formula [9]:

$N=\frac{E_{m}-I_{m} R_{m}}{K \Phi}$

Where $N$ is the speed of motor, $E_{\mathrm{m}}$ is the motor source voltage, $I_{\mathrm{m}}$ is the motor current, $R_{\mathrm{m}}$ is the motor resistance, $K$ is the constant value is depended on designing factors, and $\Phi$ is the total flux in air gap per pole. 
According to the (1), speed of motor is dependent on mechanical and electrical parameters but in robotic area we are able to change motor speed by changing in an accessible parameter is source voltage. In robotic applications, motors speed control is required to obtain various rotation methods and robot trajectory control. Different DC motor drives are designed for various applications to control and monitor motor behavior during a task. Several voltage control techniques [10] are used to control DC motors such as SCRarmature, Chopper-armature, and pulse-width modulation. In this robot, PWM technique is employed to control speed of robot's wheel using faster switching frequency.

For driving robot's motor in two directions, forward and reverse, H-bridge circuit [11] is required. The H-bridge circuit includes four switches as shown in Fig. 2.

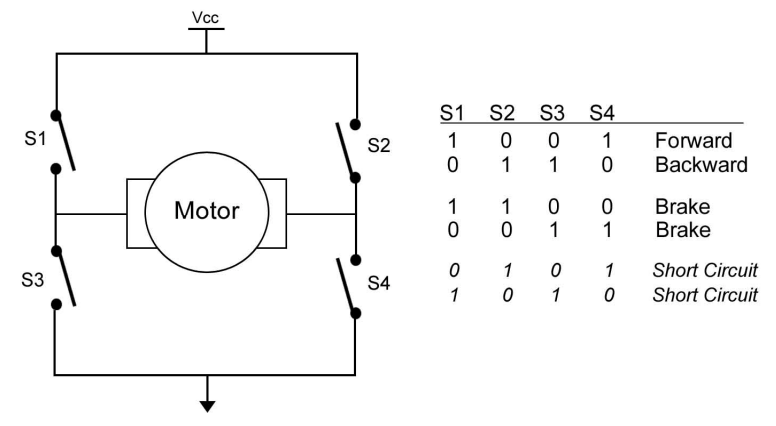

Fig.2 H-Bridge circuit for DC motor control

Commands of switches are generated by the processing unit according required motion direction. These switches are setup with various components such as Relay, Transistor, and MOSFET. To obtain high-frequency switching, semiconductor switches are required. MOSFET can provide more current with less heating in high-frequency switching rates. Si9988 is an integrated H-bridge circuit with $200 \mathrm{kHz}$ switching rate which is used for driving AMiR motors. It is able to provide continuous $650 \mathrm{~mA}$ current is suitable as the motors driver.

\section{B. Controller Unit}

A microcontroller as the main processor is deployed to generate high frequency PWM signals for robot locomotion controlling. Also, it manages other modules of AMiR such as sensors and communication. Utilized microcontroller works in $8 \mathrm{MHz}$ clock source that is generated by internal $\mathrm{RC}$ oscillator. Fig. 3 shows the main board of AMiR which equipped to the Si9988 H-bridge IC. The PWM output of microcontroller connected to H-bridge IC directly. The processor provides fast PWM signals continuously and robot motion is controlled closely by the processor. Two PWM registers value are changed in each trajectory simultaneously.

\section{Pulse Width Modulation}

One of popular techniques for reducing voltage and motor driving is pulse-width modulation [10] that uses duty-cycle principle. In this technique output power is average of on and off times. The average voltage is calculated by the following formula:

$$
V_{\text {avg }}=\frac{t_{\text {ON }}}{t_{\text {ON }}+t_{\text {OFF }}} V_{\text {in }}
$$

PWM could be a flexible, reliable and fast solution while we can limit the output current and trace the reference setpoint as fast as possible. It is very low power-lossy in comparison with other techniques. The utilized microcontroller supports two independent PWM timers. Each counter has a separate 10 bit register to define setpoints. The PWM duty-cycle can be between 0 and 1023 . When the number is increased, pulse-width will be decreased and average output voltage will be decreased. Fig. 4 illustrates voltage versus duty-cycle diagram for five different pulse-width percentages $(0 \%, 25 \%, 50 \%, 75 \%$, and $100 \%$ ). This diagram is the general voltage-average rule for PWM technique but in AMiR motors control this values are changed because of motion direction as described in trajectory control section.

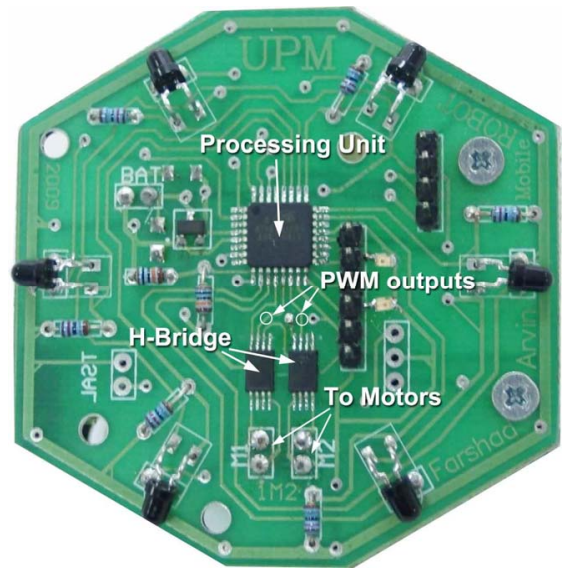

Fig.3 Main board of AMiR (processor and motor driver modules)

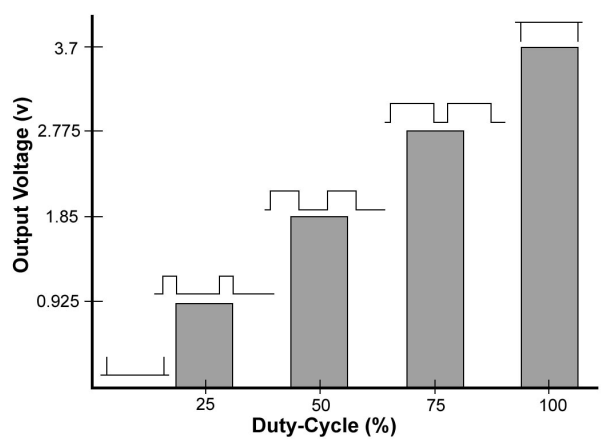

Fig.4 Output voltage as a function of duty-cycle

Speed of motor has a directly related to power voltage [9] as shown in (1). Therefore, robot motion speed is controllable with PWM register value without any complex calculation and it is a ratio of employed motor maximum speed.

For assuming motors' speed, maximum pulse values $(100 \%)$ never used to motion speed because of the recovery time of power switches. This time in MOSFET switches is very short. In addition, for stopping motors, we never turn off the motor because of next starting current, so large size 
zero pulses are used to stop motion. In this case, the circuit is alive, so we do not face inrush currents at start up and of course the motor has power to stand at the position but still we have switching and consequently switching is power loss. For long time stop, it is not suitable method because of power consuming.

In proposed technique, $96 \%$ duty-cycle is selected for maximum speed and $6 \%$ duty-cycle is used for stopping.

\section{Robot trajectory control}

DC motors and speed controlling techniques and also driving methods were described in previous section. This background is required to solve a significant problem in robotic applications which is trajectory estimation $[6,12]$. Reliable motors control functions and suitable motors and also drives are required to have a smooth trajectory. Differential drive with a caster wheel configuration is utilized by $\mathrm{AMiR}$ and a reliable motion control of differential drive technique is employed [13, 14]. The velocity of AMiR is calculated by the following formula where $d_{w}$ is the diameter of AMiR wheel is $32 \mathrm{~mm}$ and $N$ is the speed of the motor which is 85 RPM.

$$
V_{\text {robot }}=\pi d_{w} N \cong 854 \mathrm{~cm} / \mathrm{min}
$$

In this section, two common movement method, direct and rotation movement are described.

\section{1) Direct movement}

AMiR enables to have two different directions motion, forward and reverse. As the described PWM technique, speed and direction control are very simple approach which are solved with software programming. 10 bit register as the PWM set-point value can control the AMiR speed and also motion direction. The PWM register values are between 0 and 1023. The values between 0 and 512 are used to forward movement and the values between 512 and 1023 are used to backward movement. So, we can control AMiR rotation speed between 0 and maximum speed with changing in this values.

\section{2) Rotation}

The rotation and motion estimation is required for robot localization. Two different methods are used for AMiR rotation about a point: i) rotation with large diameter as shown in Fig. 5 (a) which the axis of rotation is out of AMiR area, and ii) turning in place as shown in Fig. 5 (b) which the axis of rotation is in center of AMiR's wheelbase.

In large diameter rotation, both motors rotational speed is different and calculated by the following formula:

$N_{L}=\frac{2 R+W}{2 R-W} N_{R}$

Where $N_{L}$ is the speed of left motor and $N_{R}$ is the speed of right motor. $W$ is the wheelbase width and $R$ is the radius of rotation. Also, the radius of rotation is calculated by the following formula:

$R=\frac{W}{2}\left(\frac{N_{L}+N_{R}}{N_{L}-N_{R}}\right)$
This equation is relative between wheelbase width and wheels' velocities.

For turning in place, both motors run in similar speed with different direction. The speed of wheels is calculated by (3). The time required to $n$ rotate is calculated by:

$$
\begin{aligned}
& x=n \pi W \\
& t=\frac{x}{v}, \quad(3,6) \Rightarrow t=\frac{n \cdot W}{N \cdot d_{w}}
\end{aligned}
$$
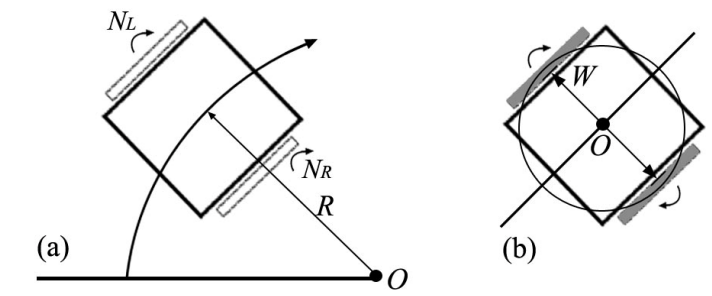

(b)

Fig.5 AMiR rotation types, (a) the axis of rotation is out of AMiR area, (b) turning in place

\section{RESUlTS AND DisCUSSION}

In this section, results of DC motor controller signals and AMiR behaviors are discussed. The result of several experiments in PWM signals output and AMiR rotation show the feasibility of implemented technique for motion controlling. Fig. 6 illustrates the ratio of PWM register value to output voltage.

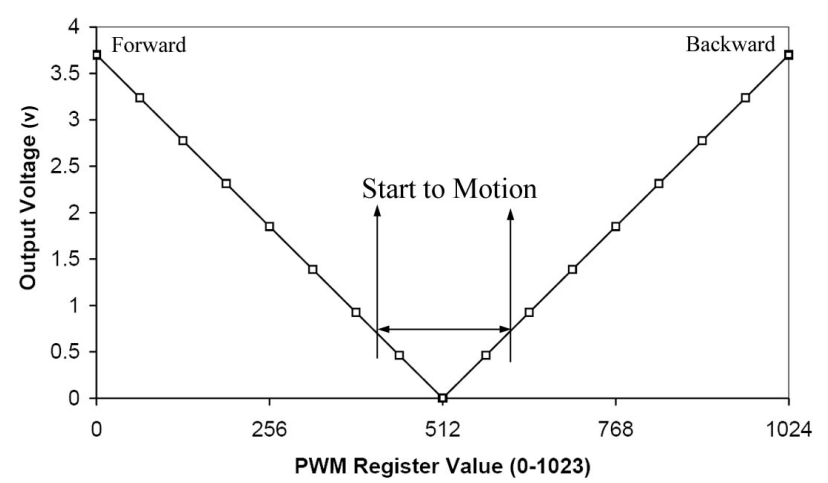

Fig.6 The output voltage indicating the speed and direction of the robot as a function of the PWM register value

Fig. 7 is captured with digital oscilloscope and it illustrates generated command wave forms for motors driving in different motion behaviors. Fig. 7 (a) is the output signals for driving motors in maximum speed, Fig. 7 (b) is the control signal for half speed forwarding, and Fig. 7 (c) is the $50 \%$ duty-cycle for stopping state which average voltage will be zero. Second experiment is the robot rotation with two different functions, turning in place and large size rotation radius (>wheelbase/2). For turning in place, two motors use similar duty-cycle with different directions. Fig. 7 (d) illustrates PWM signals for turning in place function with maximum speed and Fig. 7 (e) is the rotation in place with half speed. The rotation time for different angles is 
calculated by (7). Fig. 7 (f) illustrates command signals to obtain rotation with $R=10 \mathrm{~cm}$. One of the motors runs in maximum speed and another motor speed calculated by (4).

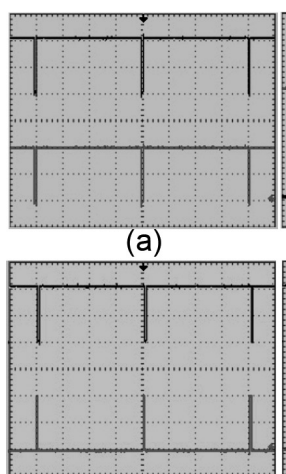

(d)

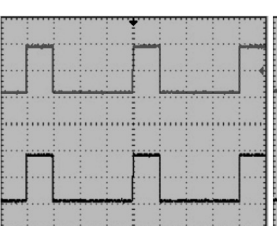

(b)

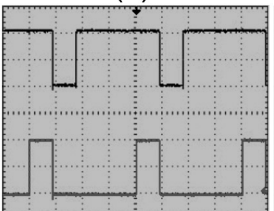

(e)

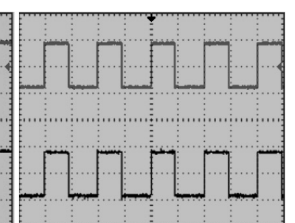

(c)

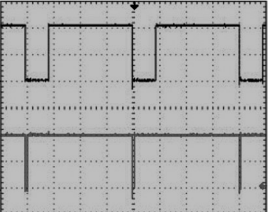

(f)
Fig.7 PWM signals generated by AMiR, (a) maximum speed, (b) half speed, (c) stop, (d) (e) turning in place, (f) rotation with large diameter

Fig. 8 illustrates current of robot's motors alter to the proportion of PWM register value with $3.8 \mathrm{v}$ supply. This diagram is the result of AMiR motors current in two different experiments, motors with maximum load and without load. The power consumption of AMiR in different behaviors is calculable according to this diagram.

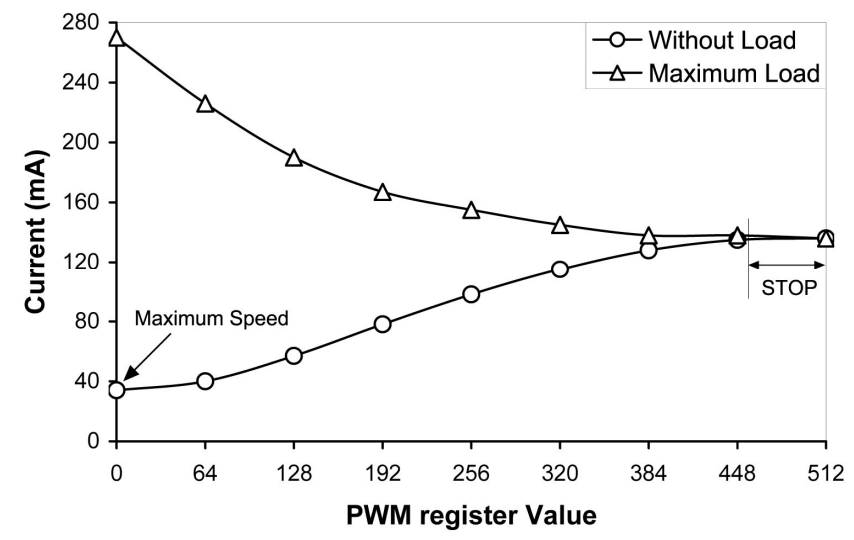

Fig.8 Current vs. PWM register value

The results of different rotations for turning in place mode are shown in Table I. The similar speed of motors (45rpm) is used for this experiment. The second column values are calculated by (7). Third column contents are the results of AMiR rotation with $t$ millisecond turning and the fourth column values are the ratio of calculated rotation angle to real result which illustrates coefficient of fault in mechanical units as shown in Fig 9. This ratio shows the additional constant gain value equal by about 0.91 which is linear const value.

Therefore, to calculate the real turning time for AMiR rotation, we have to multiply 0.91 by result of (7). This number is called coefficient of mechanical fault. Therefore, the new rotation time estimation formula is as follows:

$t=\frac{n \cdot W}{N \cdot d_{w}}=\left(0.91 \times \frac{n \times 6.5}{45 \times 3.2} \times 60\right) \mathrm{sec}$
TABLE I

EVALUATION OF AMIR PERFORMANCE WHILE TURNING IN PLACE

\begin{tabular}{lccc}
\hline \hline Rotation & $\begin{array}{c}\text { Calculated Time } \\
(\mathrm{ms})\end{array}$ & AMiR Turning & $\begin{array}{c}\text { Fault } \\
\text { Coefficient }\end{array}$ \\
\hline $45^{\circ}$ & $338 \mathrm{~ms}$ & $47^{\circ}$ & 0.95 \\
$90^{\circ}$ & $676 \mathrm{~ms}$ & $98^{\circ}$ & 0.91 \\
$135^{\circ}$ & $1014 \mathrm{~ms}$ & $148^{\circ}$ & 0.91 \\
$180^{\circ}$ & $1352 \mathrm{~ms}$ & $200^{\circ}$ & 0.90 \\
$225^{\circ}$ & $1690 \mathrm{~ms}$ & $245^{\circ}$ & 0.91 \\
$270^{\circ}$ & $2028 \mathrm{~ms}$ & $295^{\circ}$ & 0.91 \\
$315^{\circ}$ & $2366 \mathrm{~ms}$ & $342^{\circ}$ & 0.92 \\
$360^{\circ}$ & $2704 \mathrm{~ms}$ & $393^{\circ}$ & 0.91 \\
\hline \hline
\end{tabular}

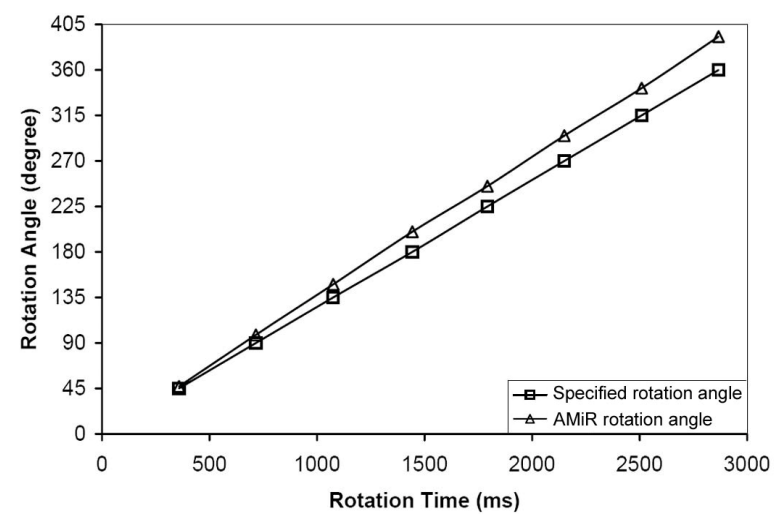

Fig.9 Comparison of AMiR actual and calculated rotation angle as a function of time

Another rotation method is the rotation with large diameter. For this method, two motors work with similar dimension and different speeds. For achieving this rotation method, a function based on (5) is defined. In this function, constant speed is selected for one of AMiR motors according to rotation direction and another motor's speed is relative to rotation radius. It is calculated by following formula:

(5) $\Rightarrow N_{2}=\frac{N_{1}\left(R-\frac{W}{2}\right)}{R+\frac{W}{2}}, R \geq W / 2$

$N_{1}=45, W=6.5 \mathrm{~cm}$

$\Rightarrow N_{2}=\frac{45 \times(R-3.25)}{R+3.25}, R>3.25 \mathrm{~cm}$

If selected $R$ is less than $W / 2(<3.25)$ then results will be negative value which shows $N_{2}$ should work reverse. A simple behavior is defined by (10) which robot must be control its motors with two different speed states as shown in Table II. Fig. 10 illustrates robot motion in this sequential behavior.

TABLE II

DEFINED BEHAVIORS FOR LARGE DIAMETER ROTATION

\begin{tabular}{ccccc}
\hline \hline Seq. & $\begin{array}{c}\text { Diameter } \\
(\mathrm{cm})\end{array}$ & $\begin{array}{c}\mathrm{N} 1 \\
(\mathrm{rpm})\end{array}$ & $\begin{array}{c}\mathrm{N} 2 \\
(\mathrm{rpm})\end{array}$ & $\begin{array}{c}\text { Rotation Time } \\
(\mathrm{ms})\end{array}$ \\
\hline 1 & 40 & 45 & 32.5 & 4166 \\
2 & 20 & 23 & 45 & 2083 \\
\hline \hline
\end{tabular}




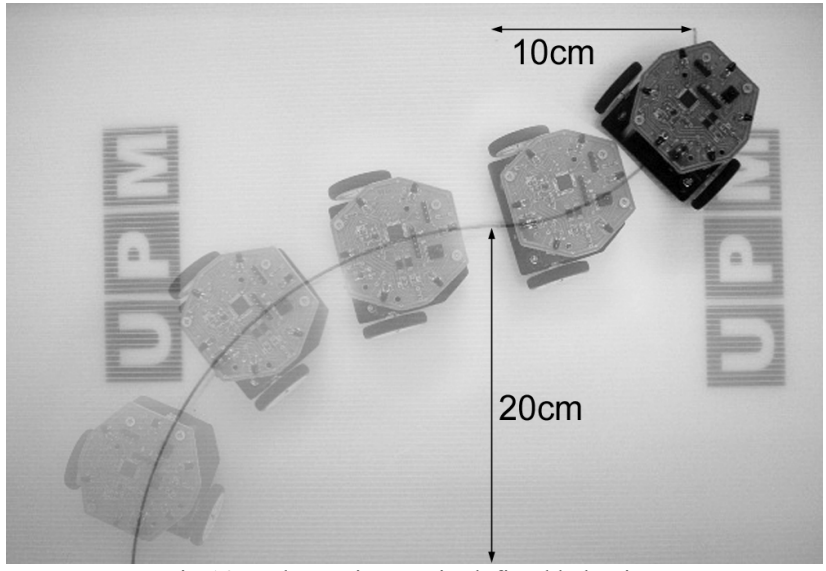

Fig.10 Robot trajectory in defined behaviors

\section{CONCLUSION}

In this paper a trajectory control technique based on pulsewidth modulation is presented. This technique is tested in autonomous miniature robot (AMiR) and the gotten results demonstrate reliability of this technique to use for differential-drive mobile robots. Several motion and rotation methods are discussed and mathematical equations' results are tested with AMiR. The PWM signals are generated by the microcontroller which they were captured with a digital oscilloscope and discussed in results section. This technique with small changes in equations is usable to employ in all mobile robots which need different speed level and smooth trajectory.

\section{REFERENCES}

[1] Appin Knowledge Solutions, Robotics, Infinty Science Press LLC, 2007, pp.156-179.

[2] J. Borenstein, "Control and kinematic design of multi-degree-of freedom mobile robots with compliant linkage", IEEE Transaction on Robotics and Autonomous, vol. 11, 1995, pp. 21-35.

[3] Y. Chung, C. Park, F. Harashima, "A position control differential drive wheeled mobile robot",IEEE Transaction on Industrial Electronic, Vol.48, 2001, pp.853-863.

[4] Hamidreza Chitsaz ,Steven M. LaValle ,Devin J. Balkcom ,Matthew T. Mason, "Minimum Wheel-Rotation Paths for Differential-Drive Mobile Robots", IEEE International Conference on Robotics Automation, ICRA'06, 2006, pp. 1616-1623.

[5] S. Rezaei, J. Guivant, E. M. Nebot, " Car-like robot path following in large unstructured environments", IEEE/RSJ International Conference on Intelligent Robots and Systems, vol 3, 2003, pp.2468- 2473.

[6] Y. Liu, X. Wu, J. Zhu, J. Jae Lew, "Omni-directional mobile robot controller design by trajectory linearization", Proceedings of the American Control Conference, vol.4, 2003, pp.3423-3428.

[7] Devin J. Balkcom, Paritosh A. Kavathekar, and Matthew T. Mason, "Time-optimal Trajectories for an Omni-directional Vehicle", The International Journal of Robotics Research, vol. 25, 2006, pp. 985 999.

[8] Dieter Foxy, Wolfram Burgardy, and Sebastian Thrun, "Controlling Synchro-drive Robots with the Dynamic Window Approach to Collision Avoidance", IEEE/RSJ International Conference on Intelligent Robots and Systems, vol. 3, 1996, pp.1280-1287.

[9] R. Michaelis, R. Mutti, J. Overmyer, O. Taylor, All About Motors an NJATC Textbook, Thomson, 2004, pp.202-211.

[10] Robert W. Erikson, M. Dragan, Fundamentals of Power Electronics, Springer, Eddition 2, 2001.

[11] John Iovine, Robots, androids, and animatrons, McGraw-Hill, 2001

[12] S. Fleury, P. Soueres, J. P. Laumond, R. Chatila, "Primitives for smoothing mobile robot trajectories", IEEE Transactions on Robotics and Automation, vol.11, 1995, pp.441-448.

[13] Richard Valentine, Motor Control Electronics Handbook, McGrawHill Handbook, Chapter 3, 1998.

[14] Paul Scherz, Practical Electronics for Invertors, McGraw-Hill Professional, 2006, pp.812. 